\title{
Un nouvel agenda de la SFMCP !
}

\section{New calendar for SFMCP!}

\section{Bouysset}

(C) Springer-Verlag France 2013

À quoi sert l'activité de notre société ?

La communauté scientifique évolue. De nos jours, meilleurs sont les résultats statistiques, meilleures sont les probabilités. Et le lecteur est rassuré sur la qualité d'une publication. Certains éléments faussent pourtant les données. Les biais de recrutement sont là et le chercheur essaie de les éliminer. Il évite de considérer les patients qui ont un droit évident à réparation. Leurs résultats sont souvent d'interprétation difficile.

Les méta-analyses regroupent des travaux disparates, parfois hétérogènes, dont les résultats d'ensemble permettent au moins d'appréhender les effets secondaires néfastes possibles d'un traitement.

Bien sûr la qualité scientifique ne peut exister sans une approche rigoureuse et des résultats vérifiés par la statistique. Mais une approche faite d'observations et de comparaisons simples restera utile pour impulser les orientations de la recherche purement scientifique. D'où l'importance de praticiens compétents et divers. Ils observent les mêmes patients sous des angles différents.

La Société Française de Médecine et de Chirurgie du Pied (SFMCP) travaille avec des praticiens de toutes origines, aux expériences diverses. En ce sens, ses travaux permettent d'approcher la « vraie vie ». L'amélioration des connaissances en pathologie du pied et de la cheville doit être transmise.

Or l'évolution est rapide. Les ostéotomies de Weil étaient presque la règle il y a 15 ans. La chirurgie percutanée de l'avant-pied devient plus fréquente actuellement.

La SFMCP œuvre pour participer à la recherche et pour la transmission des connaissances.

Merci aux praticiens, qu'ils soient issus de cette société, ou issus d'autres sociétés, pour nous aider à travailler sur tant de sujets divers.

Remercions aussi les sponsors qui nous aident, dont les éditions Springer, dans cette démarche commune. Continuons!
Nous apprenons avec tristesse le décès, à Paris, le 14 juillet 2013, de Madame le Docteur Simone Braun, épouse du Docteur Simon Braun.

La Société française de médecine et chirurgie du pied adresse toutes ses condoléances au Docteur Simon Braun, à ses enfants, ses petits-enfants et arrière-petits-enfants.

Paul Hubert Benamou

M. Bouysset

Président de la SFMCP 